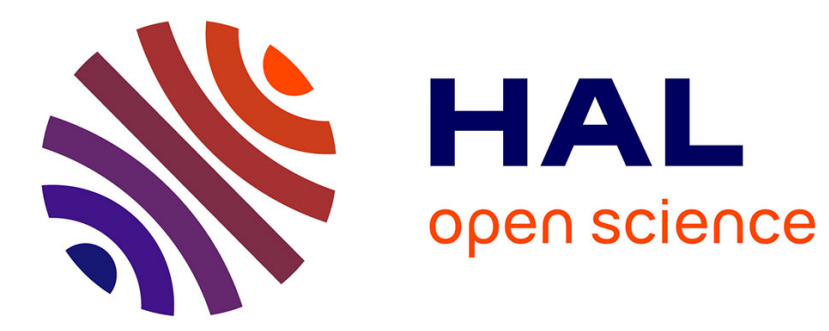

\title{
Discovery of New Gamma-Ray Pulsars with AGILE
}

\author{
A. Pellizzoni, M. Pilia, A. Possenti, A. Chen, A. Giuliani, A. Trois, P. \\ Caraveo, E. del Monte, F. Fornari, F. Fuschino, et al.
}

\section{To cite this version:}

A. Pellizzoni, M. Pilia, A. Possenti, A. Chen, A. Giuliani, et al.. Discovery of New Gamma-Ray Pulsars with AGILE. The Astrophysical journal letters, 2009, 695 (1), pp.L115-L119. 10.1088/0004637X/695/1/L115 . insu-02882253

\section{HAL Id: insu-02882253 \\ https://hal-insu.archives-ouvertes.fr/insu-02882253}

Submitted on 18 Aug 2020

HAL is a multi-disciplinary open access archive for the deposit and dissemination of scientific research documents, whether they are published or not. The documents may come from teaching and research institutions in France or abroad, or from public or private research centers.
L'archive ouverte pluridisciplinaire HAL, est destinée au dépôt et à la diffusion de documents scientifiques de niveau recherche, publiés ou non, émanant des établissements d'enseignement et de recherche français ou étrangers, des laboratoires publics ou privés. 
THIS IS AN AUTHOR-CREATED, UN-COPYEDITED VERSION OF AN ARTICLE ACCEPTED FOR PUBLICATION IN THE ASTROPHYSICAL JOURNAL LETTERS. IOP PUBLISHING LTD IS NOT RESPONSIBLE FOR ANY ERRORS OR OMISSIONS IN THIS VERSION OF THE MANUSCRIPT OR ANY VERSION DERIVED FROM IT. THE DEFINITIVE PUBLISHER AUTHENTICATED VERSION IS AVAILABLE ONLINE AT 10.1088/0004-637X/695/1/L115.

Preprint typeset using LTEX style emulateapj v. 08/13/06

\title{
DISCOVERY OF NEW GAMMA-RAY PULSARS WITH AGILE
}

A. Pellizzoni ${ }^{1}$, M. Pilia $^{1,2,3}$, A. Possenti $^{1}$, A. Chen $^{2,4}$, A. Giuliani $^{2}$, A. Trois $^{5}$, P. CaraveO $^{2}$, E. Del Monte $^{5}$, F. Fornari $^{2}$,

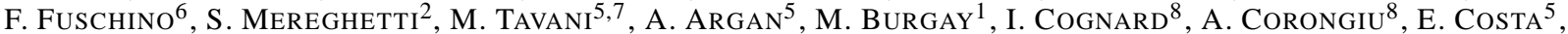
N. D’ Amico ${ }^{1}$, A. De LuCA ${ }^{2,9,10}$, P. Esposito ${ }^{2,9}$, Y. Evangelista ${ }^{5}$, M. Feroci $^{5}$, S. Johnston ${ }^{11}$, M. KRAMER $^{12}$, F. LONGO $^{13}$, M. Marisaldi ${ }^{6}$, G. Theureau ${ }^{8,14}$, P. Weltevrede ${ }^{11}$, G. Barbiellini ${ }^{13}$, F. BofFelli ${ }^{9,15}$, A. Bulgarelli ${ }^{6}$, P. W. Cattaneo ${ }^{9}$ V. $\mathrm{COCCO}^{5}$, F. D’ Ammando ${ }^{5,7}$, G. De PARIS ${ }^{5}$, G. Di CoCCO $^{6}$, I. DonNARUMma ${ }^{5}$, M. Fiorini ${ }^{2}$, T. FroYSland ${ }^{4,7}$, M. GAlli ${ }^{16}$,

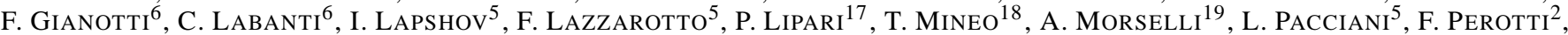

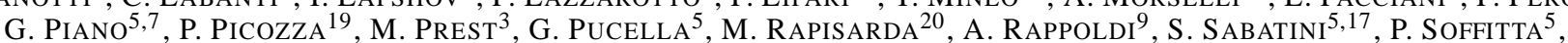
M. Trifoglio ${ }^{6}$, E. VAllazza ${ }^{13}$, S. Vercellone ${ }^{2}$, V. VitTorini ${ }^{7}$, A. Zambra ${ }^{2}$, D. Zanello ${ }^{17}$, C. Pittori $^{21}$, F. Verrecchia ${ }^{21}$, B. Preger ${ }^{21}$, P. Santolamazza ${ }^{21}$, P. Giommi ${ }^{21}$, L. SAlotti ${ }^{22}$, And G. F. Bignami ${ }^{10}$

${ }^{1}$ INAF-Osservatorio Astronomico di Cagliari, località Poggio dei Pini, strada 54, I-09012 Capoterra, Italy; apellizz@ca.astro.it 2 INAF/IASF-Milano, Via E. Bassini 15, I-20133 Milano, Italy

${ }^{3}$ Dipartimento di Fisica, Università dell'Insubria, Via Valleggio 11, I-22100 Como, Italy

${ }^{4}$ CIFS-Torino, Viale Settimio Severo 3, I-10133, Torino, Italy

${ }^{5}$ INAF/IASF-Roma, Via del Fosso del Cavaliere 100, I-00133 Roma, Italy

${ }^{6}$ INAF/IASF-Bologna, Via Gobetti 101, I-40129 Bologna, Italy

${ }^{7}$ Dipartimento di Fisica, Università "Tor Vergata", Via della Ricerca Scientifica 1, I-00133 Roma, Italy

${ }^{8}$ Laboratoire de Physique et Chimie de l'Environnement, CNRS, F-45071 Orleans, France

${ }^{9}$ INFN-Pavia, Via Bassi 6, I-27100 Pavia, Italy

${ }^{10}$ Istituto Universitario di Studi Superiori, V.le Lungo Ticino Sforza 56, 27100 Pavia, Italy

${ }^{11}$ Australia Telescope National Facility, CSIRO, P.O. Box 76, Epping NSW 1710, Australia

12 University of Manchester, Jodrell Bank Observatory, Macclesfield, Cheshire SK11 9DL, UK

${ }^{13}$ Dipartimento di Fisica, Università di Trieste and INFN-Trieste, Via Valerio 2, I-34127 Trieste, Italy

${ }^{14}$ GEPI, Observatoire de Paris, CNRS, Université Paris Diderot; Place Jules Janssen F-92190 Meudon, France

${ }^{15}$ Dipartimento di Fisica Nucleare e Teorica, Università di Pavia, Via Bassi 6, Pavia, I-27100, Italy

${ }^{16}$ ENEA-Bologna, Via Biancafarina 2521, I-40059 Medicina (BO), Italy

${ }^{17}$ INFN-Roma "La Sapienza", Piazzale A. Moro 2, I-00185 Roma, Italy

18 INAF/IASF-Palermo via U. La Malfa 153, I-90146, Palermo, Italy

${ }^{19}$ INFN-Roma "Tor Vergata", Via della Ricerca Scientifica 1, I-00133 Roma, Italy

${ }^{20}$ ENEA-Roma, Via E. Fermi 45, I-00044 Frascati (Roma), Italy

${ }^{21}$ ASI-ASDC, Via G. Galilei, I-00044 Frascati (Roma), Italy and

${ }^{22}$ ASI, Viale Liegi 26 , I-00198 Roma, Italy

Received 2009 January 08; Accepted 2009 February 27

\begin{abstract}
Using gamma-ray data collected by the Astro-rivelatore Gamma ad Immagini LEggero (AGILE) satellite over a period of almost one year (from 2007 July to 2008 June), we searched for pulsed signals from 35 potentially interesting radio pulsars, ordered according to $F_{\gamma} \propto \sqrt{\dot{E}} d^{-2}$ and for which contemporary or recent radio data were available. AGILE detected three new top-ranking nearby and Vela-like pulsars with good confidence both through timing and spatial analysis. Among the newcomers we find pulsars with very high rotational energy losses, such as the remarkable PSR B1509-58 with a magnetic field in excess of $10^{13}$ Gauss, and PSR J2229+6114 providing a reliable identification for the previously unidentified EGRET source 3EG 2227+6122. Moreover, the powerful millisecond pulsar B1821-24, in the globular cluster M28, is detected during a fraction of the observations. Four other promising gamma-ray pulsar candidates, among which is the notable J2043+2740 with an age in excess of 1 million years, show a possible detection in the timing analysis only and deserve confirmation.
\end{abstract}

Subject headings: gamma rays: observations - pulsars: general — pulsars: individual (PSR J2229+6114, PSR B1509-58, PSR B1821-24) — stars: neutron

\section{INTRODUCTION}

Although the bulk of the electromagnetic energy output of spin-powered pulsars is typically expected above $10 \mathrm{MeV}$, only $\sim 0.5 \%$ of the radio pulsar population has been clearly identified in the gamma-ray domain (Thompson 2004; Pellizzoni et al. 2008; Halpern et al. 2008). Such meager harvest is to be ascribed, most probably, to the relatively low sensitivity of gamma-ray instruments with respect to radio and $\mathrm{X}$-ray telescopes. Poor gamma-ray pulsar statistics has been a major difficulty in assessing the dominant mechanism which channels pulsar rotational energy into high-energy emission as well as understanding the sites where charged particles are accelerated.

The dominant mechanisms and sites of the emission most probably depend on the rotational period $P$ and magnetic field $B$ of the neutron stars, with the millisecond pulsars (e.g. Harding et al. 2005) behaving differently from the "classical", higher magnetic field ones. Population synthesis simulations, featuring comprehensive statistical analysis of diverse models of emission geometry and beaming, yielded very different numbers of radio-loud and radio-quiet pulsars potentially detectable as gamma-ray emitters (see e.g., Gonthier et al. 
2007). However, such simulations are poorly constrained by the source sample currently available and only a much larger sample of gamma-ray pulsars could help discriminating different emission models.

Astro-rivelatore Gamma ad Immagini LEggero (AGILE) is a scientific mission of the Italian Space Agency, dedicated to high-energy astrophysics (Tavani et al. 2008) launched on 2007 April 23. The sensitivity to photons with energy in the range $30 \mathrm{MeV}$ to $30 \mathrm{GeV}$ of the AGILE Gamma-Ray Imaging Detector (GRID; Prest et al. 2003; Barbiellini et al. 2001) together with a time tagging accuracy of a few $\mu$ s and a very large field of view ( $\gtrsim 60^{\circ}$ radius) all make AGILE perfectly suited for the detection of the time signature of new gammaray pulsars.

The large field of view of AGILE allows long uninterrupted observations and simultaneous monitoring of tens of nearby radio pulsars belonging to the "gamma-ray pulsar region" of the $P-\dot{P}$ diagram characterized by $B>2 \times 10^{11} \mathrm{G}$ and spindown energy $\dot{E}_{\text {rot }}>1.3 \times 10^{33} \mathrm{erg} \mathrm{s}^{-1}$ (Pellizzoni et al. 2004).

At variance with the behavior at soft X-ray energies, where the emission is proportional to the rotational energy loss over the squared distance factor $\left(F_{\mathrm{X}} \propto \dot{E} d^{-2}\right.$, where $d$ is pulsar distance; e.g. Possenti et al. 2002), the expected gamma-ray flux of radio pulsars is directly correlated to the GoldreichJulian current/open field line voltage (Cheng \& Ruderman 1980; Harding 1981). It can be estimated according to the law $F_{\gamma} \propto \sqrt{\dot{E}} d^{-2}$ (Kanbach 2002; Arons 1996), which is known to reasonably fit EGRET pulsars. The large dispersion of such fit (probably due to different beaming fractions) provides the minimum/maximum normalization values, allowing a worst/best case approach for the gamma-ray flux estimates. Following such an approach, we have built a sample of $\sim 100$ radio-loud pulsars which are likely to be above the AGILE sensitivity threshold $\left(F_{\min }>2 \times 10^{-8} \mathrm{ph} \mathrm{cm}^{-2} \mathrm{~s}^{-1}\right.$ at $E>100$ $\mathrm{MeV})$. Of course, we are fully aware that the actual number of detections will depend upon the emission model geometry and efficiency.

Top-ranking targets with poor AGILE exposure, wellknown pulsars already deeply investigated at the epoch of EGRET observations, and sources reserved to the AGILE Guest Observer Program were excluded from our list which encompasses 35 gamma-ray pulsar candidates. ${ }^{1}$ In this Letter we present results about isolated pulsars only. The five pulsars in binary systems of our sample will be discussed in subsequent works.

Since gamma-ray pulsar detection must start from an at least approximate knowledge of recent pulsar ephemeris, a dedicated pulsar radio monitoring campaign has been undertaken. Conservatively, we present here timing analysis results and detection claims only for pulsars having simultaneous radio ephemeris for the whole relevant AGILE observing epochs. Our campaign will continue throughout the AGILE mission for most of the targets (accordingly to their visibility), using two telescopes (Jodrell Bank and Nançay) of the European Pulsar Timing Array (EPTA), as well as the Parkes

\footnotetext{
${ }^{1}$ In ranking order: J0737-3039*, J1833-1034, J1744-1134, J1740+1000, J1747-2958, J2043+2740，J1730-2304，J1513-5908，J1524-5625，J19093744*, J1357-6429, J1531-5610, J1809-1917, J1617-5055, J1803-2137, J1801-2451，J0940-5428，J1549-4848，J1718-3825，J1824-2452，J17303350, J0900-3144*, J1420-6048, J1739-3023, J1751-2857*, J1804-2717*, J2229+6114, J1105-6107, J1721-2457, J1124-5916, J1722-3712, J1740 3015, J1823-3106, J1745-3040, J1016-5857 (the asterisk indicates binary systems). See http://agile.asdc.asi.it for details about AGILE Data Policy and AGILE Team Targets List.
}

radio telescope of The Australia Telescope National Facility (ATNF). Details about radio data analysis procedure in use for AGILE are described in Pellizzoni et al. (2008). Timing solutions for the detected pulsars can be requested by contacting the corresponding author.

\section{AGILE OBSERVATIONS AND DATA ANALYSIS}

Pulsar data were collected since the early phases of the mission. Timing observations suitable for pulsed signal analysis started in 2007 July (at orbit 1146) after engineering tests on the payload. In this Letter, we analyze data collected up to 2008 June 30.

AGILE pointings consist of long exposures, typically lasting 10-30 days, slightly drifting $\left(\lesssim 1^{\circ}\right.$ day $\left.^{-1}\right)$ from the starting direction in order to match solar panel illumination constraints. The AGILE total exposure (computed with the GRID scientific analysis task AG_ExpmapGen) for the 11 month data span considered is typically $\lesssim 10^{9} \mathrm{~cm}^{2}$ s for each target.

Data screening, particle background filtering and event direction and energy reconstruction were performed by the $A G$ ILE Standard Analysis Pipeline (BUILD-15). We adopted the AGILE event extraction criteria and timing procedures calibrated and optimized with the observations of known gammaray pulsars as described in Pellizzoni et al. (2008). In particular, we performed our timing analysis looking for pulsed signals using both $E>50 \mathrm{MeV}$ G class events (i.e. events identified with good confidence as photons) and the combination of $\mathrm{G}+\mathrm{L}$ events ( $\mathrm{L}$ events are significantly contaminated by particle background) collected within $60^{\circ}$ from the center of GRID field of view and with an extraction radius around pulsar positions optimized as a function of photon energy according to the point-spread function (PSF) and background level of AGILE. For noisy observations in confused regions (e.g. for $\mathrm{J} 2229+6114$ ), an extraction radius of $\sim 2^{\circ}$ (well below $E>50 \mathrm{MeV} \mathrm{PSF}^{2}$ ) was used to optimize the signal-tonoise ratio. Source position accuracy is $<0.5$ at present level of targets exposure.

Given the measured AGILE's time tagging accuracy of $<200 \mu$ s and the good radio monitoring (i.e., valid epoch range, adequate number of time of arrivals (ToAs)) available for the majority of our targets, the most significant pulsed signal detection is typically expected within the errors of the radio ephemeris frequency values. In particular, we performed standard epoch folding ${ }^{3}$ over a frequency range defined according to $3 \sigma$ errors of radio ephemeris.

The frequency resolution is $0.5 / T_{d s}$ (where $T_{d s}$ is the time span relative to the coverage of each target) and we oversampled it by a factor of $10\left(0.05 / T_{d s}\right.$ is the frequency step of our grid). Since the AGILE useful data span for pulsar detection is typically of the order of few months, implying a period search resolution of $<10^{-10} \mathrm{~s}$, the resulting number of independent period search trials turns out to be few tens while no independent trials on period derivatives are needed.

Pearson's $\chi^{2}$ statistics is applied to the 10-bin folded pulse profiles resulting from each set of spin parameters, yielding the probabilities (weighted for the number of trials performed on the data set) of sampling a uniform distribution, assessing

\footnotetext{
2 The on-axis AGILE PSF $67 \%$ containment radius is $\sim 10^{\circ}$ for $E=50$ $\mathrm{MeV}, \sim 5^{\circ}$ for $E=100 \mathrm{MeV}$, and $\sim 0.5$ for $E=1 \mathrm{GeV}$.

${ }^{3}$ When adequate radio observations covering the time span of the gammaray observations are available (i.e. where the WAVE terms are included in TEMPO2 ephemeris files; (Hobbs et al. 2004, 2006; Edwards et al. 2006)), we account also for the pulsar timing noise in the folding procedure as reported in Pellizzoni et al. (2008).
} 
TABLE 1

EMISSION PARAMETERS OF THE SEVEN PULSARS DISCUSSED IN TEXT (FIRMLY DETECTED GAMMA-RAY PULSARS ARE REPORTED IN THE UPPER PART OF THE TABLE)

\begin{tabular}{|c|c|c|c|c|c|c|c|c|c|c|c|c|}
\hline PSR Name & $\begin{array}{r}\text { G.Lon. } \\
\text { deg }\end{array}$ & $\begin{array}{r}\text { G.Lat. } \\
\text { deg }\end{array}$ & $\begin{array}{r}P \\
\mathrm{~ms}\end{array}$ & $\begin{array}{l}\tau^{\mathrm{a}} \\
\mathrm{yr}\end{array}$ & $\begin{array}{r}D^{\mathrm{a}} \\
\mathrm{kpc}\end{array}$ & $\begin{array}{r}\log \dot{E} \\
\operatorname{erg~s}^{-1}\end{array}$ & $\chi_{\text {red }}^{2}\left(N_{s t}\right)^{\mathrm{b}}$ & $\sigma_{\text {time }}{ }^{\mathrm{c}}$ & $\sigma_{\text {space }}^{\mathrm{d}}$ & $F_{\gamma}{ }^{\mathrm{d}}$ & $\begin{array}{l}\log L_{\gamma}^{\mathrm{e}} \\
\mathrm{erg} \mathrm{s}^{-1}\end{array}$ & $L_{\gamma} / \dot{E}$ \\
\hline $\mathrm{J} 2229+6114$ & 106.65 & 2.95 & 51.6 & $1.0 \times 10^{4}$ & 12.0 & 37.35 & $6.0(36)$ & 5.0 & 7.5 & $26 \pm 4$ & 35.36 & 0.01 \\
\hline J1513-5908 & 320.32 & -1.16 & 150.7 & $1.6 \times 10^{3}$ & 5.8 & 37.25 & $4.2(3)$ & 4.0 & 6.4 & $34 \pm 6^{\mathrm{f}}$ & 35.04 & 0.006 \\
\hline $\bar{J} 1016-5857$ & 284.08 & -1.88 & 107.4 & $2.1 \times 10^{4}$ & 9.3 & 36.41 & $6.0(69)$ & 4.8 & 12.3 & $62 \pm 6^{\mathrm{r}}$ & 35.71 & 0.2 \\
\hline J1357-6429 & 309.92 & -2.51 & 166.1 & $7.3 \times 10^{3}$ & 4.1 & 36.49 & $5.2(7)$ & 4.7 & 1.8 & $<14$ & $<34.35$ & $<0.007$ \\
\hline $\mathrm{J} 2043+2740$ & 70.61 & -9.15 & 96.1 & $1.2 \times 10^{6}$ & 1.1 & 34.75 & $4.1(1)$ & 4.2 & 0.6 & $<6$ & $<32.84$ & $<0.01$ \\
\hline
\end{tabular}

${ }^{a}$ Dispersion measure distance and characteristic age obtained from ATNF Pulsar Catalog (http://www.atnf.csiro.au/research/pulsar/psrcat/; Manchester et al. 2005). For J2229+6114, Halpern et al. (2001) derived a much shorter alternative distance of $3 \mathrm{kpc}$ from X-ray absorption.

'bearson's $\chi^{2}$ statistics applied to the 10 -bin, $E>50 \mathrm{MeV}$ folded pulse profiles $\left(N_{s t}\right.$ is the number of steps over period search grid, see text).

${ }^{\mathrm{c}}$ Pulsed emission detection significance (weighted accounting for $N_{s t}$ ).

${ }^{\mathrm{d}}$ Likelihood analysis detection significance and flux (or $2 \sigma$ upper limit) in units of $10^{-8} \mathrm{ph} \mathrm{cm}^{-2} \mathrm{~s}^{-1}(E>100 \mathrm{MeV})$, obtainined by search for a source near the pulsar direction.

${ }^{\mathrm{e}} E>100 \mathrm{MeV}$ luminosity assuming traditional $1 \mathrm{sr}$ beam emission and photon index 2.0 (for $\mathrm{J} 2229+6114$ the measured photon index of 2.2 has been used).

${ }^{\mathrm{f}}$ Possibly multiple sources. Source confusion may affect flux and significance.

the significance of the pulsed signal (sinusoidal or not). In fact, applying this statistics to the well-known EGRET pulsars (Pellizzoni et al. 2008), we obtained a perfect match between the best period resulting from AGILE gamma-ray data and the period predicted by the radio ephemeris with discrepancies comparable to the period search resolution. Furthermore, we verified our timing results also applying bin-independent parameter-free statistics as the $Z_{n}^{2}$ test (Buccheri et al. 1983) and the $H$-test (de Jager et al. 1989) that are typically more sensitive than $\chi^{2}$ tests for the search of sinusoidal pulses. In particular, the use of the $H$-test is suggested if no a priori information about the light curve shape is available as in our case.

First, for each target we searched for pulsed signals using the whole available data span. Later, each observation block was analyzed to check for possible flux and/or pulse profile variability.

We also performed a preliminary maximum likelihood analysis (ALIKE task) on the AGILE data for the regions containing our targets in order to exploit the instrument's imaging capabilities to assess gamma-ray source parameters. Here, we focus on timing analysis leaving detailed source positioning, flux and (phase-resolved) spectral analysis for future papers when higher counts photon statistics on each target will be available and instrument effective area calibrations will be consolidated.

\section{NEW GAMMA-RAY PULSARS}

Table 1 lists the emission parameters of the seven pulsars discussed in this Letter. The resulting radio-aligned light curves are plotted in Figure 1 where for each pulsar the actual bin size and energy range has been adjusted according to the available statistics and light-curve structure. We note that in all cases radio and gamma-ray timing results are compatible, with the highest significance frequency detected in gamma rays within the errors of the radio ephemeris value, considering also the period search resolution. Examples of the exploration of much larger frequency search grids are shown in Figures 2 and 3 for PSR J2229+6114, for which the best gamma signal is within $1 \sigma$ from the radio peak, and for PSR J1824-2452, for which the radio-gamma frequency discrepancy is comparable with the gamma-ray period search resolution. Both $\chi^{2}$ statistics and $Z_{n}^{2} / H$-test provide comparable detection significances, except for PSR J2229+6114. The $Z_{n}^{2}$ test applied to this pulsar provides slighly better results than the other statistics $\left(Z_{1}^{2}=39.5, Z_{2}^{2}=45.9\right.$ corresponding to an $\sim 6 \sigma$ detection). Furthermore, we verified that our analysis procedure (potentially affected by instrument-related systematic errors and biases in events extraction criteria) does not produce fake detections at a significance level above $3 \sigma$ when the radio-ephemeris are applied to randomly extracted AGILE data.

Four targets are also firmly detected by the likelihood spatial analysis. It is not surprising that spatial detection be missing for weaker targets at the present AGILE exposure level. In high-background regions of the Galactic plane, our pulsed emission search sensitivity can be better than our spatial analysis sensitivity. Furthermore, timing analysis can be applied to the full AGILE event list (i.e. $\mathrm{G}+\mathrm{L}$ event classes extending up to $60^{\circ}$ from the center of the field of view), while, currently, spatial analysis is only fully calibrated for the $\mathrm{G}$ class events detected within $40^{\circ}$ from the center of the field of view.

However, some of the non-detections in spatial analysis are, indeed, puzzling. According to Figure 1, PSR J2043+2740 has a pulsed flux and light curve very similar to PSR J15135908 (note that both are based on $\mathrm{G}+\mathrm{L}$ events). Moreover, $\mathrm{J} 2043+2740$ is out of the Galactic plane, in a region well exposed and with low diffuse emission. Thus, it should have been detected by the image analysis more easily than J15135908, which sits in a higher background region, unless, of course, they have very different spectra.

Pulsars in Table 1 are ranked according to their overall detection significance. Pulsars firmly detected both through timing and spatial analyses are placed above the line. Pulsars detected through timing analysis alone await longer simultaneous gamma-ray and radio observations for confirmation and reliable luminosity estimation.

In Table 1 , the most significant detection is PSR J2229+6114 for which AGILE detected pulsed emission (radio/gamma-ray periods discrepancy $\lesssim 10^{-11}$ s, Figure 2) and pinpointed the most likely position $(l=106.86$, $b=2.94$ ) to $\sim 0.2$ from the radio pulsar. Our detection provides a reliable identification for the previously unidentified 


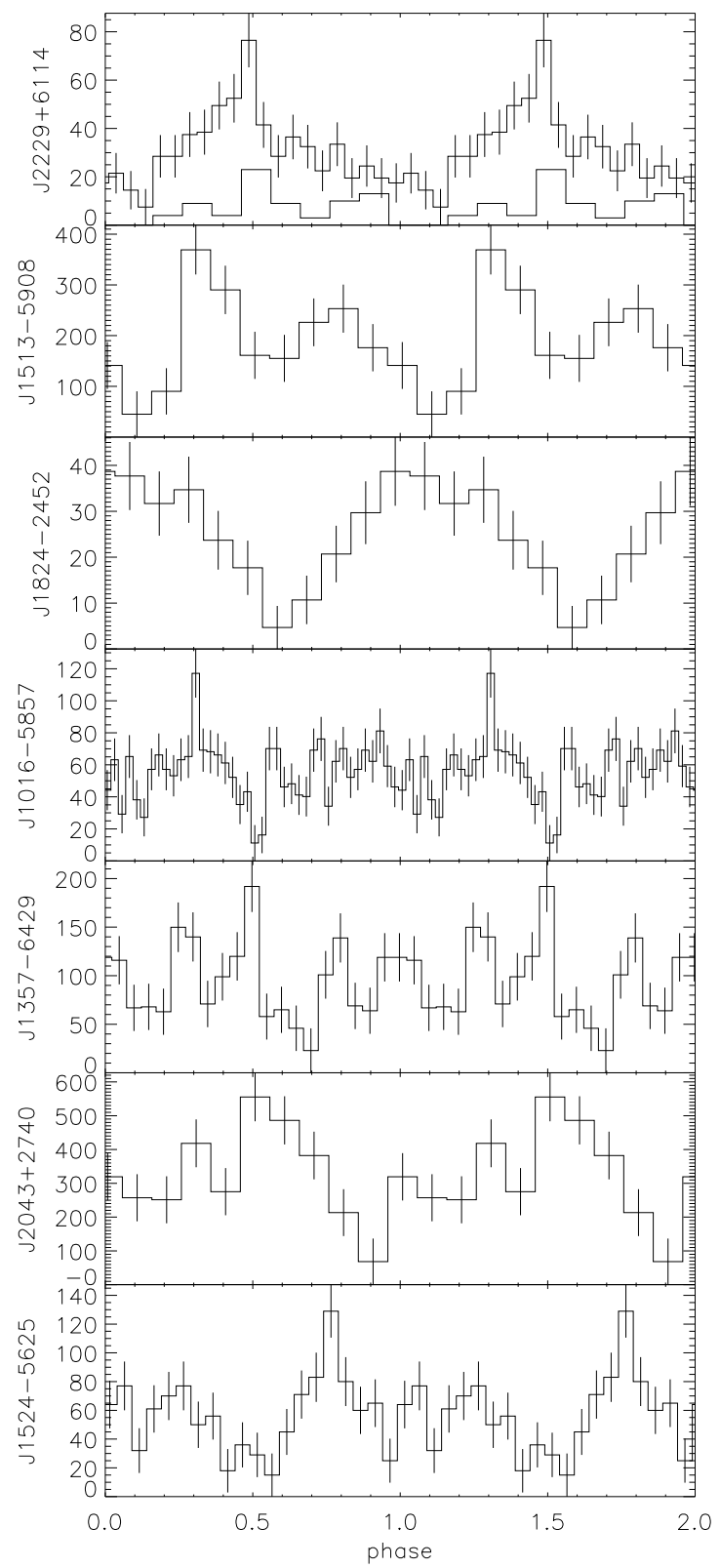

FIG. 1.- Background-subtracted $E>100 \mathrm{MeV}$ folded pulse profiles (except for the $E>50 \mathrm{MeV} \mathrm{J1357-6429}$ light curve) of the pulsars shown in Table 1. Events extraction radius is optimized as a function of energy (see the text). All pulse profiles are obtained using $\mathrm{G}$ event class only except for PSR J1513+5908 and PSR J2043+2740 that have higher counts statistics because detections are obtained including $\mathrm{G}+\mathrm{L}$ event class. For PSR J2229+6114 the histogram of the $\sim 75(\mathrm{G}+\mathrm{L})$ events with $E>1 \mathrm{GeV}$ is also shown. Absolute timing is performed for each target: the main radio peak $(1.4 \mathrm{GHz})$ corresponds to phase 0 . Possible fluctuations of the dispersion measure over the considered time interval are not expected to significantly affect phasing results, given the time resolution of the available gamma-ray light curves.

EGRET source 3EG 2227+6122 $(l=106.53, b=3.18)$. The AGILE source position, pulsed flux, and photon index ( 2.2) are consistent with the EGRET values (Hartman et al. 1999). The gamma-ray light curve of this pulsar (detected up to over $1 \mathrm{GeV}$ ), featuring just one prominent peak shifted $\sim$ $180^{\circ}$ in phase from the radio main peak, is shown in Figure 1. It is worth noting that assuming a distance of $\sim 3 \mathrm{kpc}$

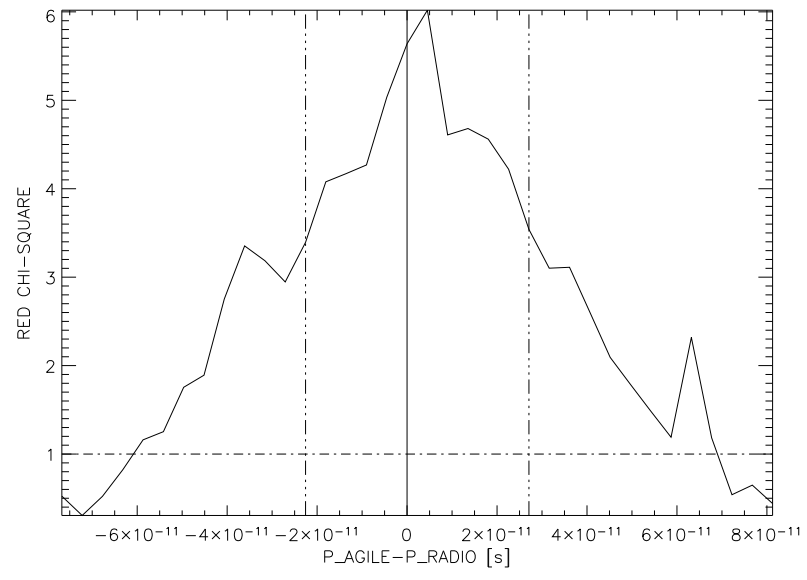

FIG. 2.- PSR J2229+6114: gamma-ray period search result (period trials versus $\chi^{2}$ Pearson statistics). The radio period (vertical line at $\left.P_{\text {AGILE }}-P_{\text {RADIO }}=0\right)$ is $51.64101208(3) \mathrm{ms}(\mathrm{PEPOCH}=54575.5856 \mathrm{MJD})$. The most significant gamma-ray pulse profile is obtained for $P_{\text {AGILE }}^{\text {Best }}=$ $51.641012085(2)$, a value within the $1 \sigma$ error on the radio period (vertical dashed lines).

inferred by X-ray observations (Halpern et al. 2001) and isotropic emission, the pulsar gamma-ray efficiency would be $\sim 0.5$, a factor of 20 higher than that quoted in Table 1 . In modern pulsar beaming models (in particular high-altitude models) the assumption of isotropy in luminosity calculation could be a better approximation in most cases, implying that efficiencies should be increased by roughly an order of magnitude from those based on the commonly assumed $1 \mathrm{sr}$ beam (Watters et al. 2008). Anyway, precise efficiency measurements await for a better assessment of pulsar distance, flux, and beam geometry.

PSR J1513-5908 (B1509-58) was detected by COMPTEL in the 1-10 MeV range, while EGRET reported (Fierro 1996) only marginal evidence for a weak $<4 \sigma$ source at $\sim 1^{\circ}$ from the radio position, with a pulsed emission upper limit of $<58 \times 10^{-8} \mathrm{ph} \mathrm{cm}^{-2} \mathrm{~s}^{-1}$. The AGILE discovery of pulsed emission from PSR B1509-58 relies also on a $6.4 \sigma$ detection of a gamma-ray source (possibly multiple sources) at $\lesssim 0.4$ from the radio position. The main gamma-ray peak at phase $\sim 0.35$ in the AGILE light curve is aligned with the soft gamma-ray peak seen by COMPTEL in the $0.75-30 \mathrm{MeV}$ band $(0.35 \pm 0.03$; Kuiper et al. 1999$)$ slightly trailing the hard X-rays single peaked light curves (Saito et al. 1997; Rots et al. 1998). A second possibile peak in the AGILE light-curve at $\sim 0.85$ could have correspondence with the marginal feature seen in the 10-30 MeV COMPTEL light curve. The COMPTEL sharp spectral break between 10 and $30 \mathrm{MeV}$ (Kuiper et al. 1999) is confirmed by our AGILE point at $100 \mathrm{MeV}$. Our data imply a softening of the photon index from $\sim 1.7$ to $\sim 2.5$ going from tens to hundreds of MeV. Such a lowenergy break, compared to the more common $\mathrm{GeV}$ spectral break in gamma-ray pulsars, could be the telltale signature of the photon splitting process inducing significant e.m. cascade attenuation due to the strong magnetic polar field (greater than $10^{13} \mathrm{G}$ ) of this pulsar (e.g. Harding et al. 1997).

At variance with all the other targets, the millisecond pulsar J1824-2452 in the Globular Cluster M28 was detected by AGILE with good significance (greater than $4 \sigma$ ) and perfect radio-gamma periods match, only in the time interval 5433954344 MJD (Figure 3). The main radio peak at $1.4 \mathrm{GHz}$ is coincident with the broad single peak seen in gamma rays. 


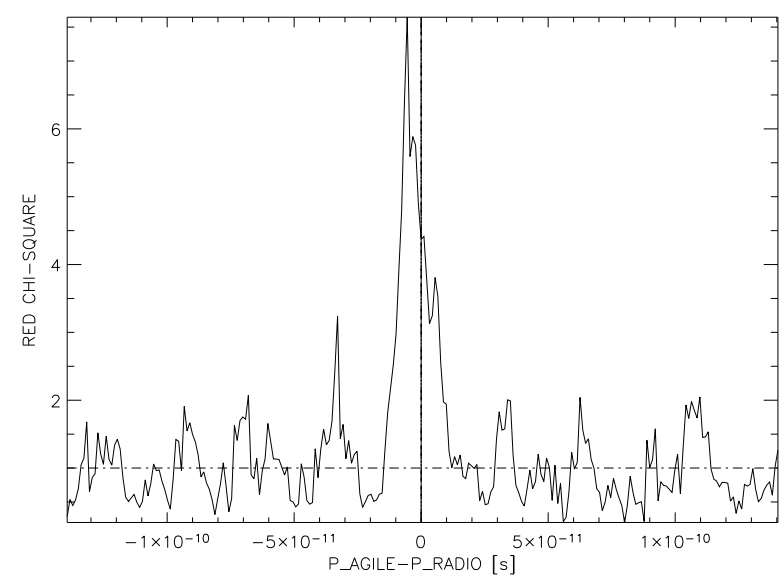

FIG. 3.- PSR J1824-2452: gamma-ray period search result. The radio period is $3.0543151208713(1) \mathrm{ms}(\mathrm{PEPOCH}=51468 \mathrm{MJD})$. The most significant gamma-ray pulse profile is obtained for $P_{\text {AGILE }}^{\text {Best }}=3.05431511(1) \mathrm{ms}$. The corresponding radio-gamma periods discrepancy $\lesssim 10^{-11} \mathrm{~s}$ is comparable to the period search resolution in the considered data span (54339-54344 MJD).

Only marginal detection was obtained integrating other observations with comparable exposure or the whole data span. Noise fluctuations could possibly explain the apparent variability. Alternatively, although its gamma-ray efficiency and high stability of spin parameters are compatible with rotationpowered emission, some additional mechanism disturbing the neutron star magnetosphere in the dense cluster environment could be invoked to explain the variable gamma-ray phenomenology of this peculiar pulsar. AGILE timing failures at submillisecond level in some observations (mimicking source variability) cannot be excluded. However, this seems unlikely, since we verified both timing accuracy and stability at $\sim 200$ $\mu$ s level with Vela pulsar observations. Confirmation of this tantalizing result about physical variability will rest on longer monitoring campaigns.

PSR J1016-5857 stands out in Table 1 for its very high efficiency in converting rotational energy loss into high-energy gamma rays. This may be ascribed to distance uncertainties, as happened to be the case for the recently discovered PSR J2021+3651 (Halpern et al. 2008) for which the distance derived from the dispersion measure is certainly overestimated. Furthermore, we note that the position $(l=284.47$, $b=-0.94)$ and flux of the AGILE gamma-ray source is only marginally compatible with 3EG 1013-5915, for which multiple associations were proposed (Hartman et al. 1999). The region, originally covered by the gamma-ray source 2CG 28400, discovered by COS-B (Swanenburg et al. 1981), is very complex. Other nearby sources, and in particular pulsar PSR J1016-5819 (not belonging to our sample), could significantly contribute to the high flux observed by AGILE in this region.

Of the remaining three pulsars, detected only through timing analysis, the most notable is certainly PSR J2043+2740 which, with an age in excess of one million years, would be the oldest ordinary (nonrecycled) pulsar seen in the gammaray domain. The precise measurements of its flux will yield its luminosity and thus its efficiency, a parameter of paramount importance for the understanding of gamma-ray emission as a function of the pulsar age.

According to their timing and spatial analysis outcomes, light-curve profiles, and radio-gamma phasing upshots, PSR J2229+6114, J1513-5908 and J1824-2452 can be considered rather solid detections. If the detection of J10165857, J2043+2740, J1357-6429, and J1524-5625 will also be firmly established, our seven object sample, together with the detection of J2021+3651 (Halpern et al. 2008), would imply that gamma-ray emission is a common feature of luminous radio-loud pulsars, be they young or old. Indeed, our list encompasses the second youngest (J1513-5908) and by far the oldest nonrecycled pulsar (J2043+2740) detected at gammaray energies. The gamma-ray pulsar search and the improvement of AGILE timing procedures requiring unprecedented long integrations is an ongoing effort, in close collaboration with the radio observers. We trust that the new gamma-ray pulsar detections presented here will trigger multiwavelength observations (e.g. in X-rays) simultaneous to the continuously improving AGILE and Fermi (formerly GLAST) exposures on these targets. The availability of high-resolution light curves and their precise multiwavelength phasing will pave the way to improve pulsar models as well as our understanding of the pulsar population as a whole.

We thank the referee for her/his useful recommendations and comments. The Parkes radio telescope is part of the Australia Telescope, which is funded by the Commonwealth of Australia for operations as a National Facility managed by CSIRO. We thank Jules Halpern and Fernando Camilo for useful discussions and contributions on gamma-ray pulsar timing.

\section{REFERENCES}

Arons, J. 1996, A\&AS, 120, 49

Barbiellini, G., et al. 2001, in AIP Conf. 587, Gamma-Ray Astrophysics, ed.

S. Ritz, N. Gehrels, \& C. R. Shrader (Melville, NY: AIP), 754

Buccheri, R., et al. 1983, A\&A, 128, 245

Cheng, A. F. \& Ruderman, M. A. 1980, ApJ, 235, 576

de Jager, O. C., Raubenheimer, B. C., \& Swanepoel, J. W. H. 1989, A\&A, 221,180

Edwards, R. T., Hobbs, G. B., \& Manchester, R. N. 2006, MNRAS, 372, 1549

Fierro, J. M. 1996, PhD Thesis, Stanford University

Gonthier, P. L., Story, S. A., Clow, B. D., \& Harding, A. K. 2007, Ap\&SS, 309,245

Halpern, J. P., Camilo, F., Gotthelf, E. V., Helfand, D. J., Kramer, M., Lyne, A. G., Leighly, K. M., \& Eracleous, M. 2001, ApJ, 552, L125

Halpern, J. P., et al. 2008, ApJ, 688, L33

Harding, A. K. 1981, ApJ, 245, 267

Harding, A. K., Baring, M. G., \& Gonthier, P. L. 1997, ApJ, 476, 246

Harding, A. K., Usov, V. V., \& Muslimov, A. G. 2005, ApJ, 622, 531

Hartman, R. C., et al. 1999, ApJS, 123, 79

Hobbs, G. B., Edwards, R. T., \& Manchester, R. N. 2006, MNRAS, 369, 655

Hobbs, G., Lyne, A. G., Kramer, M., Martin, C. E., \& Jordan, C. 2004, MNRAS, 353, 1311
Kanbach, G. 2002, in MPE Report 278, Proceedings of the 270th WEHeraeus Seminar on Neutron Stars, Pulsars, and Supernova Remnants, ed. W. Becker, H. Lesch, \& J. Trümper (Garching: MPI), 91

Kuiper, L., Hermsen, W., Krijger, J. M., Bennett, K., Carramiñana, A., Schönfelder, V., Bailes, M., \& Manchester, R. N. 1999, A\&A, 351, 119 Manchester, R. N., Hobbs, G. B., Teoh, A., \& Hobbs, M. 2005, AJ, 129, 1993

Pellizzoni, A., Chen, A., Conti, M., Giuliani, A., Mereghetti, S., Tavani, M., \& Vercellone, S. 2004, Advances in Space Research, 33, 625

Pellizzoni, A., et al. 2009, ApJ, 691, 1618

Possenti, A., Cerutti, R., Colpi, M., \& Mereghetti, S. 2002, A\&A, 387, 993

Prest, M., Barbiellini, G., Bordignon, G., Fedel, G., Liello, F., Longo, F., Pontoni, C., \& Vallazza, E. 2003, NIMPR A, 501, 280

Rots, A. H., et al. 1998, ApJ, 501, 749

Saito, Y., Kawai, N., Kamae, T., Shibata, S., Dotani, T., \& Kulkarni, S. R. 1997, ApJ, 477, L37

Swanenburg, B. N., et al. 1981, ApJ, 243, L69

Tavani, M., et al. 2008, A\&A, in press (arXiv:0807.4254)

Thompson, D. J. 2004, in Astrophysics and Space Science Library, Vol. 304, Cosmic Gamma-Ray Sources, ed. K. S. Cheng \& G. E. Romero (Dordrecht: Kluwer), 149

Watters, K. P., Romani, R. W., Weltevrede, P., \& Johnston, S. 2009, ApJ, in press 